\title{
Syntheses of gold and silver dichroic nanoparticles; looking at the Lycurgus cup colors
}

\author{
${ }^{1}$ Department of BioNanoTechnology, Wageningen University, PO Box 8038, 6700, EK Wageningen, The Netherlands, E-mail: \\ vittorio.saggiomo@wur.nl.https://orcid.org/0000-0001-7196-602X.
}

\begin{abstract}
:
Metallic nanoparticles are small particles, with dimensions of less than $100 \mathrm{~nm}$, which have unique characteristics. For example, the color of a nanoparticle solution is given by the surface plasmon resonance, in contrast to only absorbance of a molecule. Metallic nanoparticles have been used in the antiquity for the production of colored glass. Many of the medieval cathedral red windows are in fact, stained with gold nanoparticles. However, presenting just a red solution to a class and explaining that the solution contains gold nanoparticles can be disappointing as the red color can be obtained in many different ways without the need of nanoparticles. Something peculiar, like dichroism, can, on the other hand, shine the interest of an observer to the "nanoworld". Dichroic nanoparticles show different colors when illuminated from the front (scattering) or from the back (transmission). This effect is unique for nanoparticles and nanostructures in general and can generate the "WOW" effect in class or during public demonstrations. In this article we present a simple synthesis of dichroic silver and gold nanoparticles. Solutions of such nanoparticles are a stunning visual media for demonstrations, outreach and engaging audience in the "nano"world.
\end{abstract}

Keywords: demonstration, dichroism, gold nanoparticle, silver nanoparticle

DOI: 10.1515/cti-2019-0011

\section{Introduction}

Metallic nanoparticles are nano-objects with dimensions of less than $100 \mathrm{~nm}$. They are used in many different fields nowadays, from nanomedicine as diagnostics or therapeutic agents, to antibacterial, to coloring agents. The history of metallic nanoparticles is very old, and the presence of nanoparticles, used as coloring agent, has been discovered in ancient Roman artifacts from the fourth century. Pottery and glass from the Middle Ages to the Renaissance also show presence of gold nanoparticles (AuNP) in their matrix. Interestingly, the color given by the metallic nanoparticles is due to the "surface plasmon resonance" which is characteristic for nanoparticles and it does not fade away with time. Pottery stained with gold nanoparticles, for example, still show a deep red color even after being exposed to the environment for more than 100 years. On the contrary, statues of the same age which were colored with organic compounds, are white nowadays because the organic compounds have been degraded over time. Due to the multiple applications of metallic nanoparticles and their peculiar characteristics it is important to (a) teach students about them and (b) engage a broad public to spike their interest in nano-research.

Demonstrating the presence and properties of nanoparticles is not an easy task. Most of the times, the demonstrator simply shows a red solution presenting it as a dispersion of gold nanoparticles (Faraday, 1857; Zhang, Wang, \& Xu, 2015). The same demonstration can be done with silver nanoparticles, which show different colors depending on the sizes and shapes of the particles (Huang \& Xu, 2010; Orbaek, McHale, \& Barron, 2015). Thus, the audience is only presented with colored solutions which, by eye, are indistinguishable from those with any other organic dye or inorganic pigment, except for the Tyndall effect.

\section{Dichroism and the Lycurgus Cup}

One peculiarity of nanoparticles, however, can be exploited to provoke a "WOW moment" in the audience: the dichroic filter effect (Figure 1). A dichroic material shows two different colors whether it is illuminated from 
the front (reflection) or from the back (transmission). In almost all the introductory lectures and books about nanoparticles, a classic example of dichroism is shown: the Lycurgus cup (Freestone, Meeks, Sax, \& Higgitt, 2007; Braslavsky, 2009, p. 293). This cup from the Roman Empire ( $4^{\text {th }}$ century A.D.) is the main example of a dichroic filter, showing a greenish color when illuminated from the front and a purple color when illuminated from the back. The cup is nowadays exposed at the British Museum in London where it is admired by thousands of visitors every day. The dichroic effect of the Lycurgus cup has puzzled scientists for long time and it was only later discovered to be caused by the size, shape and composition of the (gold and silver) nanoparticles embedded in the glass (Barber \& Freestone, 1990; Brill, 1965). This effect is visually stunning and it would be excellent for showing some of the properties of nanotechnology.

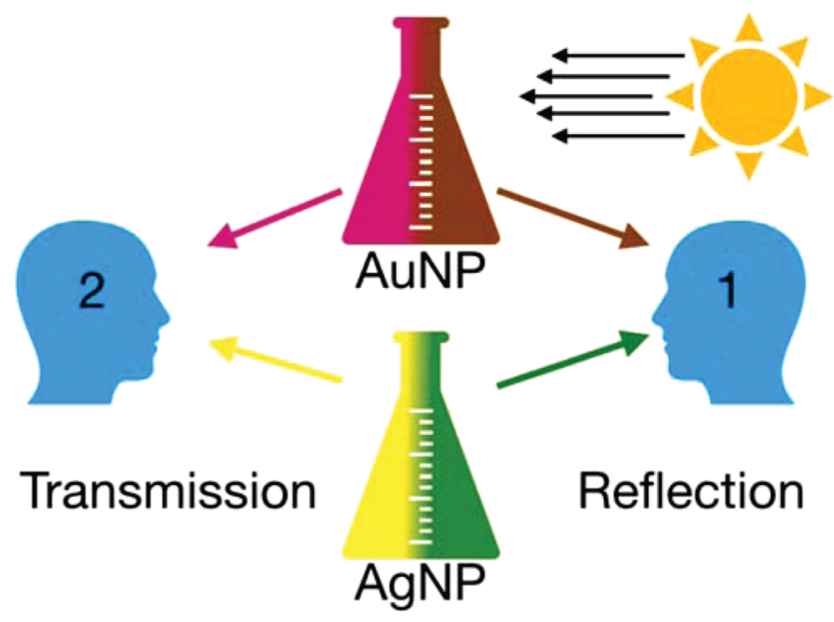

Figure 1: Dichroic nanoparticle solutions show two different colors depending on the incident light. Observer 1 will see the two solutions of gold and silver nanoparticles as brown and green respectively, while observer 2 will see them as violet and yellow.

Recently we have recreated this similar effect by 3D printing a nanocomposite material in which dichroic nanoparticles were embedded (Kool, Bunschoten, Velders, \& Saggiomo, 2019).

Here, we show how to synthesize two different kinds of dichroic nanoparticles in a simple and fast way: gold nanoparticles (AuNP) which show a brown/violet transition, and silver nanoparticles (AgNP) which show a green/yellow effect. The synthesis is fast and can be done, by a demonstrator or university students, in front of an audience in about $10 \mathrm{~min}$; the dichroic solutions are stable over time and can be stored and brought to classes or used for outreach purposes. The demonstrator, using these nanoparticles, can explain concepts such as reductive synthesis of nanoparticles (Turkevich, Stevenson, \& Hillier, 1951), plasmon resonance colors (Kristensen et al., 2016), and scattering (Mie theory, Rayleigh scattering) (Huang \& El-Sayed, 2010).

Reductive synthesis is one of the most common methods of synthesizing metallic nanoparticles; plasmon resonance is an intrinsic property of nanoparticles and it is the reason why we see colors of nanoparticles. Scattering of light on nanomaterials is, for example the reflective colors of butterfly or beetles.

\section{Demonstration}

This article is focused on demonstration not only to a general public but also for demonstration and hands-on from upper secondary to freshman. The synthesis can be performed by a demonstrator in front of a class or general public, but also directly by students (high school or university) provided they are done in a laboratory setting. The nanoparticle syntheses described here has been used for two years without any hiccups in the "BioNanoTechnology: an introduction" course, where students themselves synthesize and characterize (DLS, TEM, and UV-VIS) dichoric nanoparticles in parallel to standard nanoparticles.

\section{Procedure}

\section{Dichroic AuNP}

The synthesis of the dichroic gold nanoparticles is a variation on the "Turkevich"-method for the synthesis of gold nanoparticles (Ji et al., 2007). The preparation of a $100 \mathrm{~mm}\left(100 \mathrm{mg}\right.$ in $2.5 \mathrm{~mL}$ ) stock solution of $\mathrm{HAuCl}_{4}$ 
and a $34 \mathrm{~mm}(100 \mathrm{mg}$ per $10 \mathrm{~mL})$ stock solution of trisodium citrate dihydrate, prior to the demonstration, is advisable. Be careful not to use metal objects (like spatulas) while handling $\mathrm{HAuCl}_{4}$, as this will immediately reduce the gold, rendering it unsuitable for the synthesis of nanoparticles.

The experiment starts by preparing a $100 \mathrm{~mL} 0.25 \mathrm{mM} \mathrm{HAuCl}_{4}$ solution in a $250 \mathrm{~mL}$ Erlenmeyer flask, by adding $250 \mu \mathrm{L}$ of the $100 \mathrm{~mm} \mathrm{HAuCl}_{4}$ stock solution to $100 \mathrm{~mL}$ of distilled water. The gold solution is then heated on a heating plate. Once the solution is boiling, $500 \mu \mathrm{L}$ of the $34 \mathrm{~mm}$ citrate stock solution is added in one shot and the solution is left boiling on the heating plate.

After approximately $1 \mathrm{~min}$, the solution will change color from faintly yellow to blue/black. After another 5-10 min, the solution will change to purple/orange, indicating that dichroic gold nanoparticles have been formed. At this point, a white light can be used to show the different colors in transmission and reflection. Once cooled down, the solution of nanoparticles can be stored at room temperature for at least 8 months without losing the dichroic property. The particles tend to sediment over time; however, shaking the liquid will restore the effect.

For the success of the experiment it is important to keep both the concentration of the gold precursor, as well as the ratio between $\mathrm{Au}$ and citrate, constant. When the concentration or the ratio significantly deviate from the mentioned values, the effect is not visible. Step by step instructions are given in the notes for instructor.

\section{Dichroic AgNP}

Dichroic AgNP were obtained by a reductive synthesis of silver nitrate to AgNP by $\mathrm{NaBH}_{4}$ in the absence of a capping agent and by adding the capping agent PolyVinylPyrrolidone (PVP K30) directly afterwards.

A solution of PVP (K30; MW 40 kDa) was prepared prior to the demonstration, by dissolving $1.5 \mathrm{~g}$ (37.5 $\mu \mathrm{mol}) \mathrm{PVP}$ in $60 \mathrm{~mL}$ distilled water. To start the reaction $190 \mathrm{mg}(1.1 \mathrm{mmol}) \mathrm{AgNO}_{3}$ was dissolved in $150 \mathrm{~mL}$ distilled water in a $500 \mathrm{~mL}$ Erlenmeyer flask and the solution was stirred vigorously using a magnetic stirring bar. A freshly prepared solution of $20 \mathrm{mg}(0.5 \mathrm{mmol}) \mathrm{NaBH}_{4}$ in $30 \mathrm{~mL}$ distilled water was added to the $\mathrm{AgNO}_{3}$ solution and this was followed directly (as soon as possible) by the addition of the $60 \mathrm{~mL}$ of PVP solution.

Upon addition of $\mathrm{NaBH}_{4}$ to $\mathrm{AgNO}_{3}$, the color of the reaction mixture changes immediately to black and then, after a few seconds, to the dichroic state. The formed AgNP should be captured in this state by capping them with an excess of PVP by adding the PVP within few seconds after adding $\mathrm{NaBH}_{4}$. This timing is important, as the Ag dichroic nanoparticles are only obtained as long as the PVP is added within few seconds after the addition of $\mathrm{NaBH}_{4}$. Slight variance in colors and the intensity of the dichroic effect may be obtained depending on variables such as the stirring rate and the timing of the addition of the $\mathrm{NaBH}_{4}$ and PVP solutions; variables which are difficult to control.

The step by step instructions are given in the notes for instructor.

\section{Hazards}

\section{AuNP}

$\mathrm{HAuCl}_{4}$ causes irritation of the skin, eyes and respiratory tract. Use Personal Protective Equipment (PPE) when handling the gold salt and gold solutions. When a Chloroauric acid solution gets in contact with the skin, the proteins present in the skin will reduce the gold forming nanoparticles, thus staining the skin. The stain will remain on the skin for 2 or 3 days. It is also harmful if swallowed. Trisodium citrate is mildly irritating to the skin, eyes and respiratory tract. While handling chemicals, the instructors should wear suitable eye protection, wear gloves and work in a well-ventilated area.

\section{AgNP}

$\mathrm{AgNO}_{3}$ is an oxidizing agent and may react violently. It should not come into contact with combustible materials, strong bases and acids, or strong reducing agents. $\mathrm{AgNO}_{3}$ causes irritation of the skin, eyes and respiratory tract. Use gloves and PPE when handling the silver solution. $\mathrm{NaBH}_{4}$ is hazardous in case of skin or eye contact (irritant and corrosive), ingestion or inhalation. $\mathrm{NaBH}_{4}$ is also corrosive and flammable and should be handle with care and only in small amounts (the synthesis presented here produces $210 \mathrm{~mL}$ of dichroic solution using only $20 \mathrm{mg}$ of $\mathrm{NaBH}_{4}$ ).

PVP K30 is slightly hazardous in case of skin or eye contact, ingestion or inhalation. 


\section{Discussion}

The syntheses here described produces two distinct dichroic nanoparticles: gold (AuNP) and silver (AgNP). The dichroic effect is caused by the different sizes and shapes of the nanoparticles present in solution. This is evident when the solutions are observed with different illumination angles (Figure 2). In reflection, the AuNP solution shows a brown color, while in transmission, the solution looks violet. The AgNP solution shows a green color, while in transmission, the solution looks yellow. This effect can be presented to the audience in different ways. One approach, useful for example in case of an outreach activity where the audience is free to walk by, is to have a fixed lighting system while the observer moves around the solution (Video 1). Another approach, useful in case of classes or other kinds of audiences far away from the stage, is to move the lighting system around the solution (Video 2).

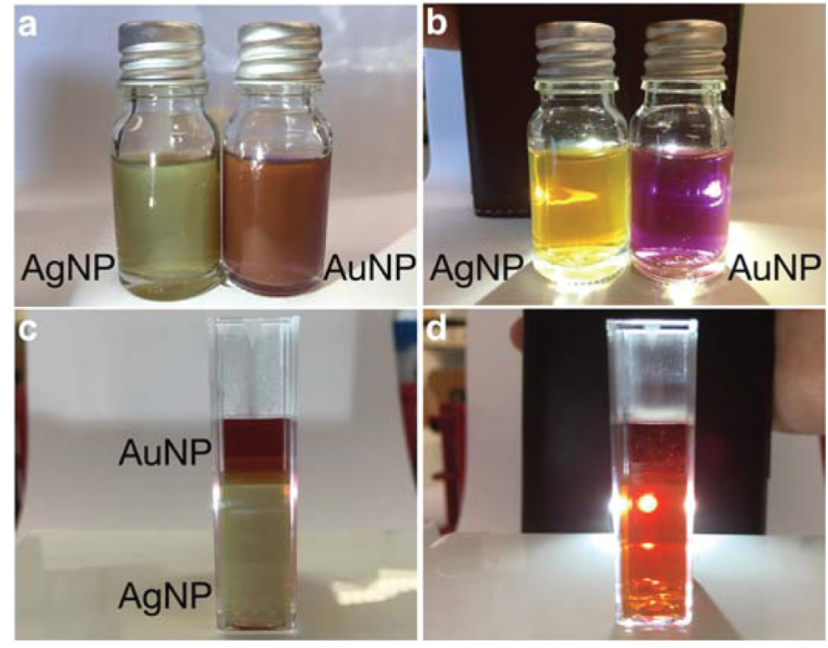

Figure 2: Silver and gold dichroic nanoparticle solutions when (a) they reflect light and (b) when they transmit light. When a cuvette with small (red) gold nanoparticles is placed behind the silver dichroic solution the result will show green in reflection (c) and orange/red in transmission (d), as the transmission is the result of the gold nanoparticles red color and the yellow color of the silver dichroic nanoparticles. Figure $\mathrm{c}-\mathrm{d}$ shows the same effect as the Lycurgus cup (green/red effect).

To demonstrate how the effect observed in the actual Lycurgus cup can be recreated a solution of small gold nanoparticles, which are red and not dichroic, can be placed behind the silver dichroic solution. This will result in observing green in reflection and red in transmission, similar to the Lycurgus cup. Mixing the two Ag and Au nanoparticle solutions together will not result in the same dichroic effect, as the two metals are not strongly capped and may undergo metal exchange.

Another effect of the dichroic nanoparticle solutions that can be used in a demonstration is the differences in transparencies. Both AuNP and AgNP solutions appear opaque in reflection and transparent in transmission. This effect can be used to hide or show text or images behind the solution. Depending on the direction of the illumination the text or image will show or remain hidden (Figure 3 and Video 3). 


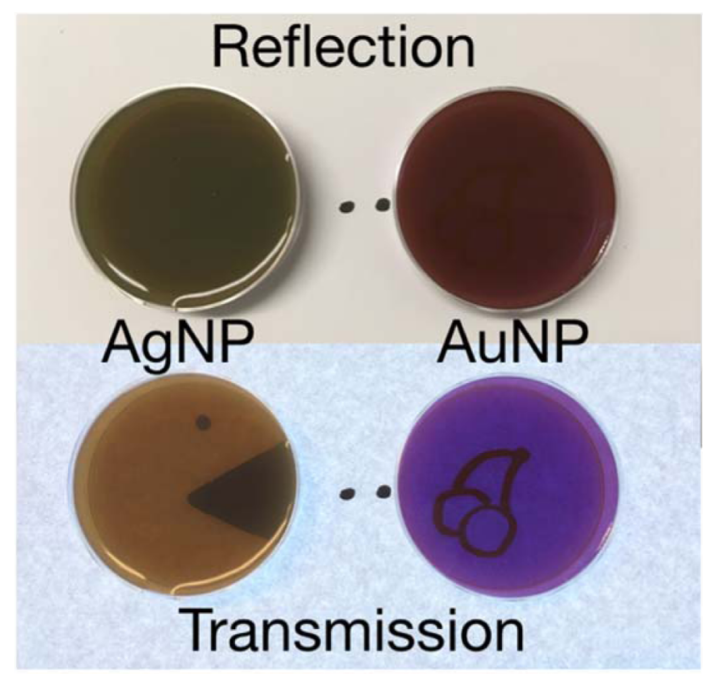

Figure 3: The dichroic effect is used to hide the images below the nanoparticle solutions. In reflection, in fact, the solutions not only present a different color from the transmittance one, but they are also opaque (top), while in transmission, the two colors are transparent (bottom).

Surprisingly, even after many decades of nanotechnology research, there are no dichroic nanoparticle syntheses aimed at teaching nanotechnology to students. The syntheses here described are fast and easy to perform compared to other synthetic methodologies present in literature (https://onlinelibrary.wiley.com/doi/abs/10.1002/adom.201200040), and can be used to showcase the beauty and power of nanotechnology.

\section{Conclusion}

We have previously shown that the formation of dichroic gold nanoparticles is due to the polydispersity of the sample synthesized with a sub optimal amount of reducing agent which favors the intraparticle ripening, forming large and non-symmetrical particles. Detailed discussion on the particle size, shape and their formation can be found in our open access article (Kool et al. 2019). We speculate similar mechanisms of formation for the dichroic silver nanoparticles.

We have shown how to prepare, in a simple and fast way, dichroic solutions of silver and gold nanoparticles. These solutions can be used in class or during outreach events to show intrinsic properties of nanoparticles, as the dichroic effect is more stunning than showing just a colored solution.

\section{Notes for instructor}

Lab instructions and videos of the dichroic nanoparticles are available as notes for instructor.

\section{References}

Barber, D., \& Freestone, I. C. (1990). An iinvestigation of the origin of the colour of the Lycurgus cup by analytical transmission electron microscopy. Archaeometry, 32, 33-45.

Braslavsky, S. (2009). Clossary of terms used in photochemistry, 3rd edition (IUPAC Recommendations 2006). Pure and Applied Chemistry, 79, 293-465.

Brill, R. H. (1965). The Chemistry of the Lycurgus Cup. In 7th International Congress of Class. Brussels.

Faraday, M. (1857). The Bakerian Lecture. - Experimental relations of gold (and other metals) to light. Philosophical Transactions of the Royal Society of London, 147,145

Freestone, I., Meeks, N., Sax, M. \& Higgitt, C. (2007). The Lycurgus Cup - A Roman nanotechnology. Cold Bulletin, 40, $270-277$.

Huang, T., \& Xu, X.-H. N. (2010). Synthesis and characterization of tunable rainbow colored colloidal silver nanoparticles using singlenanoparticle plasmonic microscopy and spectroscopy. Journal of Materials Chemistry, 20(44), 9867-9876.

Huang, X., \& El-Sayed, M. A. (2010). Gold nanoparticles: Optical properties and implementations in cancer diagnosis and photothermal therapy. Journal of Advanced Research, 1, 13-28. 
Ji, X., Song, X., Li, J., Bai, Y., Yang, W., \& Peng, X. (2007). Size control of gold nanocrystals in citrate reduction: The third role of citrate. lournal of the American Chemical Society, 129(45), 13939-13948.

Kool, L., Bunschoten, A., Velders, A. H., \& Saggiomo, V. (2019). Cold nanoparticles embedded in a polymer as a 3D-printable dichroic nanocomposite material. Beilstein Journal of Nanotechnology, 10, 442-447.

Kristensen, A., Yang, J. K. W., Bozhevolnyi, S. I., Link, S., Nordlander, P., Halas, N. J., \& Mortensen, N. A. (2016). Plasmonic colour generation. Nature Reviews Materials, 2, 16088.

Orbaek, W. A., McHale, M. M., \& Barron, R. A. (2015). Synthesis and characterization of silver nanoparticles for an undergraduate laboratory. Journal of Chemical Education, 92, 339-334.

Turkevich, J., Stevenson, P. C., \& Hillier, J. (1951). Discussions of the Faraday Society, 11, 55.

Zhang, X., Wang, Z., \& Xu, C. (2015). Demonstrationg the many possible colors of gold-supported solid nanoparticles. Journal ofChemical Education, 92, 336-338.

Supplementary Material: The online version of this article offers supplementary material (DOI: https://doi.org/10.1515/cti-2019-0011). 